\title{
Vector Risk Functions $\equiv$
}

\author{
Alejandro Balbás, Raquel Balbás and Pedro Jiménez-Guerra*
}

\begin{abstract}
The paper introduces a new notion of vector-valued risk function, a crucial notion in Actuarial and Financial Mathematics. Both deviations and expectation bounded or coherent risk measures are defined and analyzed. The relationships with both scalar and vector risk functions of previous literature are discussed, and it is pointed out that this new approach seems to appropriately integrate several preceding points of view. The framework of the study is the general setting of Banach lattices and Bochner integrable vector-valued random variables. Sub-gradient linked representation theorems and practical examples are provided.
\end{abstract}

Mathematics Subject Classification (2010). 91B30, 91G80.

Keywords. Vector risk function, representation theorem, dynamic risk measures and other examples.

\section{Introduction}

The notion of coherent measure of risk was introduced in the seminal paper by Artzner et al. (1999), and since then their work has been extended in many directions. Jouini et al. (2004) justified the use of vector random variables to represent the final wealth provided by some portfolios, as well as the use of "coherent vector-valued risk measures" to reflect risk levels. Cascos and Molchanov (2007) enlarged the set of financial applications of these new frameworks.

The interest of the approach of Jouini et al. (2004) justifies possible extensions of their discussion so as to incorporate much more practical situations. For instance, they deal with a $L_{\infty}$ space, whereas many scalar coherent risk measures are defined on a larger $L_{p}$ space (for example, $L_{1}$ is the natural

The authors thank Professor C. Zalinescu and the anonymous reviewer for their useful comments and suggestions. This research was partially supported by "Comunidad Autónoma de Madrid" (Spain), Grant S2009ESP-1685, and "Ministerio de Ciencia en Innovación" (Spain), Grant ECO2009 - 14457 - C04. The usual caveat applies.

*Corresponding author. 
space to introduce the Conditional Value at Risk). In this line, Hamel and Heyde (2010) show that the approach also makes sense in more general $L_{p}$ spaces, and Balbás and Jiménez-Guerra (2010) deal with risks and risks measures which are valued in a general Banach Lattice, rather than the classical finite-dimensional space $\mathbb{R}^{n}$.

Besides, while Artzner et al. (1999) understood their risk measures as initial capital requirements that investors and managers should provide in order to overcome negative evolutions of the market, recent literature has shown the interest of drawing on risk measures in order to address other classical topics, such as pricing and hedging issues (Nakano, 2004) or portfolio choice problems (Ogryczak and Ruszczynski, 1999, Benati, 2003, Konno et al., 2005, Rockafellar et al., 2006a, etc.). This fact has led to further studies concerning risk analysis, and the use of convex measures (Föllmer and Schied, 2002), consistent measures (Goovaerts et al. ,2004) or deviations and expectation bounded risk measures (Rockafellar et al., 2006b), amongst many other kinds of risk functions. It seems that extending the Jouini et al. (2004) analysis makes it easier to deal with the issues above under weaker restrictions.

This paper aims to present a general framework of vector risk functions. We introduce a "generalized vector risk function" as a map $\rho: L_{p}(\mu, E) \rightarrow F$, with $\mu$ being a probability and $E$ and $F$ being general Banach lattices (MeyerNieberg, 1991). According to the properties of $\rho$, we define "coherent measures", "deviations", and "expectation bounded measures". The main difference with respect to previous literature dealing with vector risk measures is that we will not deal with "set-valued functions". On the contrary $\rho(y)$ is not a complex subset of $F$ but a single element, for every $y \in L_{p}(\mu, E)$. This new approach significantly simplifies previous ones and retrieves suitable and natural properties; For instance, the simultaneous consideration of scalar deviations or coherent expectation bounded risk measures generates vector deviations or vector coherent expectation bounded risk functions. Simultaneously, we deal with a general framework, since we do not impose finite dimensions and $p$ is arbitrary within the interval $[1, \infty]$.

The outline of the paper is as follows. Section 2 introduces the general setting and those previous concepts and properties that we will need throughout the article. Section 3 introduces the generalized vector risk functions, their properties and some important relationships. Section 4 presents Representation Theorems. We have followed the idea of Rockafellar et al. (2006b), in the sense that we represent the measure $\rho$ "as an envelope of its sub-gradients", which, as long as $E$ satisfies the Radon-Nikodym property (Diestel and Uhl, 1977), are elements of $L_{q}\left(\mu, E^{*}\right) ; q$ being the conjugate of $p$ and $E^{*}$ denoting the dual space of $E$. Section 5 presents some practical examples and financial applications of vector risk functions, with special focus on dynamic risk measures. Both, scalar and vector dynamic risk measures are particular cases of the vector risk functions that we will introduce in the third section. Section 6 concludes the article. 


\section{Preliminaries and notations}

Throughout the paper, $E, F, E_{+}$and $F_{+}$will denote two Banach lattices and their non-negative cones respectively. ${ }^{1}$ Their dual Banach lattices and cones will be represented by $E^{*}, F^{*}, E_{+}^{*}$ and $F_{+}^{*}$, and $\left\langle e^{*}, e\right\rangle$ will be "the usual product" of $e^{*} \in E^{*}$ and $e \in E$. If $e_{1}, e_{2} \in E$ and $e_{1}-e_{2} \in E_{+}$then we will write $e_{1} \geq e_{2}$. Similar ideas apply if $F$ plays the role of $E$.

$\mathcal{L}(E, F)\left(\mathcal{L}_{+}(E, F)\right)$ will be the set of linear maps $\Lambda: E \rightarrow F$ that are continuous (non-negative, i.e., $\Lambda(e) \geq 0$ whenever $e \geq 0$ ). Every $\Lambda \in \mathcal{L}_{+}(E, F)$ is continuous (Meyer-Nieberg, 1991).

$(\Omega, \mathcal{F}, \mu)$ will be a probability space composed of the set $\Omega$, the $\sigma$-algebra $\mathcal{F}$ and the probability measure $\mu . p \in[1, \infty]$ and $q \in[1, \infty]$ will be conjugate values, i.e., $1 / p+1 / q=1$. If $p<\infty$ then $L_{p}(\mu, E)$ will represent the Banach space of those Bochner integrable (Diestel and Uhl, 1977) functions $y: \Omega \rightarrow E$ such that $\int_{\Omega}\|y(\omega)\|^{p} d \mu(\omega)<\infty$, endowed with the usual norm

$$
\|y\|_{p}=\left(\int_{\Omega}\|y(\omega)\|^{p} d \mu(\omega)\right)^{\frac{1}{p}} .
$$

Similarly, $L_{\infty}(\mu, E)$ will be the Banach space of $E$-valued essentially bounded and integrable functions, endowed with the norm

$$
\|y\|_{\infty}=e s s-\sup \{\|y(\omega)\| ; \omega \in \Omega\}
$$

ess - sup denoting the essential supremum. It is well known that $L_{p_{1}}(\mu, E) \subset$ $L_{p_{2}}(\mu, E)$ whenever $p_{1} \geq p_{2}$ and the natural inclusion is continuous. If $p<\infty$ and $E^{*}$ satisfies the Radon-Nikodym property then, $L_{q}\left(\mu, E^{*}\right)$ is the dual space of $L_{p}(\mu, E)$. Henceforth we will assume that $E$ and $E^{*}$ satisfy the Radon-Nikodym property (Diestel and Uhl, 1977).

If $\mathcal{G}$ denotes a sub- $\sigma$-algebra of $\mathcal{F}$ and $\mu_{\mathcal{G}}$ is the restriction of $\mu$ to $\mathcal{G}$, then $L_{p}\left(\mu_{\mathcal{G}}, E\right)$ is a closed subspace of $L_{p}(\mu, E)$. In such a case $\mathbb{E}(y \mid \mathcal{G}) \in$ $L_{p}\left(\mu_{\mathcal{G}}, E\right)$ for every $y \in L_{p}(\mu, E), \mathbb{E}(y \mid \mathcal{G})$ denoting the conditional expectation of $y$ with respect to $\mathcal{G}$.

An interesting particular example is $\mathcal{G}=\{\varnothing, \Omega\}$, in which case $L_{p}\left(\mu_{\mathcal{G}}, E\right)$ and $E$ may be identified. Indeed, if there is no confusion, for every $y_{0} \in E$ we will also represent by $y_{0}$ the constant element of $L_{p}(\mu, E)$ given by $y(\omega)=y_{0}$ a.s. Furthermore, $\mathbb{E}(y \mid \mathcal{G})$ will be represented by $\mathbb{E}(y)=\int_{\Omega} y d \mu \in E$, for every $y \in L_{p}(\mu, E)$.

\section{Generalized vector risk functions}

Definition 3.1. Every

$$
\rho: L_{p}(\mu, E) \rightarrow F
$$

\footnotetext{
${ }^{1}$ Most of the properties here stated would still hold if $E$ or $F$ were ordered Banach spaces. However we think that imposing $E$ and $F$ to be Banach lattices the exposition is significantly simplified.
} 
will be called Vector Risk Function $(V R F)$. Furthermore, $\rho$ is said to be:

a) $(\Lambda, \mathcal{G})$-Translation invariant, if $\mathcal{G}$ denotes a sub- $\sigma$-algebra of $\mathcal{F}$,

$$
\Lambda: L_{p}\left(\mu_{\mathcal{G}}, E\right) \longrightarrow F
$$

is linear and continuous, and $\rho\left(y+y_{0}\right)=\rho(y)-\Lambda\left(y_{0}\right)$ holds for every $y \in$ $L_{p}(\mu, E)$ and every $y_{0} \in L_{p}\left(\mu_{\mathcal{G}}, E\right){ }^{2}$ If $\mathcal{G}=\{\varnothing, \Omega\}$ (i.e., $L_{p}\left(\mu_{\mathcal{G}}, E\right) \approx E$ ) and $\Lambda \in L_{+}(E, F)$, then we will merely say that $\rho$ is $\Lambda$-translation invariant.

b) Positively homogeneous, if $\rho(\alpha y)=\alpha \rho(y)$ holds for every real number $\alpha>0$ and every $y \in L_{p}(\mu, E)$.

c) Sub-additive, if $\rho\left(y_{1}+y_{2}\right) \leq \rho\left(y_{1}\right)+\rho\left(y_{2}\right)$ holds for every $y_{1}, y_{2} \in$ $L_{p}(\mu, E)$.

d) Decreasing, if $\rho\left(y_{2}\right) \leq \rho\left(y_{1}\right)$ whenever $y_{1}, y_{2} \in L_{p}(\mu, E)$ and $y_{2} \geq y_{1}$ a.s.

e) $(\Lambda, \mathcal{G})$-Mean dominating, if $\mathcal{G}$ is a sub- $\sigma$-algebra of $\mathcal{F}, \Lambda: L_{p}\left(\mu_{\mathcal{G}}, E\right) \longrightarrow$ $F$ is linear and continuous, and $\rho(y) \geq-\Lambda(\mathbb{E}(y \mid \mathcal{G}))$ holds for every $y \in$ $L_{p}(\mu, E)$.

Definition 3.2. The $V R F \rho$ is said to be:

a) A $(\Lambda, \mathcal{G})$-expectation bounded risk measure, if $\mathcal{G}$ is a sub- $\sigma$-algebra of $\mathcal{F}$, $\Lambda: L_{p}\left(\mu_{\mathcal{G}}, E\right) \longrightarrow F$ is linear and continuous, and $\rho$ is $(\Lambda, \mathcal{G})$-translation invariant, positively homogeneous, sub-additive, and $(\Lambda, \mathcal{G})$-mean dominating.

b) A $(\Lambda, \mathcal{G})$-coherent risk measure, if $\mathcal{G}$ is a sub- $\sigma$-algebra of $\mathcal{F}, \Lambda: L_{p}\left(\mu_{\mathcal{G}}, E\right)$ $\longrightarrow F$ is linear and continuous, and $\rho$ is $(\Lambda, \mathcal{G})$-translation invariant, positively homogeneous, sub-additive, and decreasing.

c) A $\mathcal{G}$-deviation if $\mathcal{G}$ is a sub- $\sigma$-algebra of $\mathcal{F}$ and $\rho$ is $(\Lambda, \mathcal{G})$-expectation bounded with $\Lambda=0$.

Remark 3.3. Definition 3.2. above extends the notion of scalar coherent risk measure (Artzner et al., 1999), scalar expectation bounded risk measure (Rockafellar et al., 2006b), and scalar deviation measure (Rockafellar et al., 2006b). Scalar measures arise when $E=F=\mathbb{R}, \mathcal{G}=\{\varnothing, \Omega\}$, and $\Lambda$ is the identity map.

Proposition 3.4. Let $\rho$ be a VRF.

a) If $\rho$ is positively homogeneous then $\rho(0)=0$.

b) If $\rho$ is $(\Lambda, \mathcal{G})$-expectation bounded or $(\Lambda, \mathcal{G})$-coherent for some $(\Lambda, \mathcal{G})$ then $\rho\left(y_{0}\right)=-\Lambda\left(y_{0}\right)$ for every $y_{0} \in L_{p}\left(\mu_{\mathcal{G}}, E\right){ }^{3}$

\footnotetext{
${ }^{2}$ Following Artzner et al. (1999), if $\Lambda$ is onto we can consider that, given $y \in L_{p}(\mu, E)$, any $y_{0} \in L_{p}\left(\mu_{\mathcal{G}}, E\right)$ such that $\Lambda\left(y_{0}\right)=\rho(y)$ may be understood as a final wealth or pay-off that must be guaranteed by the initial capital requirements. Indeed, one has that

$$
\rho\left(y+y_{0}\right)=\rho(y)-\Lambda\left(y_{0}\right)=0,
$$

so the global risk vanishes with the additional wealth $y_{0}$.

${ }^{3}$ In particular, $\rho\left(y_{0}\right)=0$ for every $\mathcal{G}$-deviation $\rho$ and every $y_{0} \in L_{p}\left(\mu_{\mathcal{G}}, E\right)$. Notice that it is sufficient to impose $\rho$ to be $(\Lambda, \mathcal{G})$-translation invariant and positively homogeneous.
} 
Proof. To prove $a$ ) notice that $\rho(0)=\rho(\alpha 0)=\alpha \rho(0)$, so $\rho(0) \neq 0$ would lead to $\alpha=1$ for every positive $\alpha$.

To prove $b)$ notice that $\rho\left(y_{0}\right)=\rho\left(0+y_{0}\right)=\rho(0)-\Lambda\left(y_{0}\right)=-\Lambda\left(y_{0}\right)$.

The following result establishes the existence of a one to one mapping between deviations and expectation bounded risk measures.

Proposition 3.5. Let $\mathcal{G}$ be a sub- $\sigma$-algebra of $\mathcal{F}$ and $\Lambda: L_{p}\left(\mu_{\mathcal{G}}, E\right) \longrightarrow F$ linear and continuous. The relationship

$$
\rho \rightarrow D=\rho+\Lambda \circ \mathbb{E}(-\mid \mathcal{G})
$$

establishes a one to one correspondence between the set of $(\Lambda, \mathcal{G})$-expectation bounded risk measures and the set of $\mathcal{G}$-deviations.

Proof. If $\rho$ is a $(\Lambda, \mathcal{G})$-expectation bounded risk measure then set $D=\rho+$ $\Lambda \circ \mathbb{E}(-\mid \mathcal{G})$ and $D$ is trivially $(0, \mathcal{G})$-translation invariant, positively homogeneous and sub-additive. To show that $D$ is $(0, \mathcal{G})$-mean dominating, take $y \in L_{p}(\mu, E)$. Then,

$$
D(y)=\rho(y)+\Lambda \circ \mathbb{E}(y \mid \mathcal{G}) \geq 0
$$

because $\rho$ is $(\Lambda, \mathcal{G})$-mean dominating and $\rho(y) \geq-\Lambda \circ \mathbb{E}(y \mid \mathcal{G})$.

Conversely, suppose that $D$ is a deviation and set $\rho=D-\Lambda \circ \mathbb{E}(-\mid \mathcal{G}) . \rho$ is clearly $(\Lambda, \mathcal{G})$-translation invariant, positively homogeneous and sub-additive. To show that $\rho$ is $(\Lambda, \mathcal{G})$-mean dominating, take $y \in L_{p}(\mu, E)$, and one has that

$$
\rho(y)=D(y)-\Lambda \circ \mathbb{E}(y \mid \mathcal{G}) \geq-\Lambda \circ \mathbb{E}(y \mid \mathcal{G})
$$

because $D(y) \geq 0$.

\section{Representation theorems}

Artzner et al. (1999) and Jouini et al. (2004) stated Representation Theorems of "their coherent risk measures" (scalar and vector, respectively) by using duality properties and $\mu$-continuous finitely or $\sigma$-finitely additive measures on the measurable space $(\Omega, \mathcal{F})$. The extensions of Hamel and Heyde $(2010)$ and Balbás and Jiménez-Guerra (2010) also led to analogous (but more general) Representation Theorems. However, Rockafellar et al. (2006b) represented "their (real-valued) expectation bounded risk measures" by using $L_{2}(\mu, \mathbb{R})$, which may be identified with its dual space. Here we draw on the duality $\left(L_{q}\left(\mu, E^{*}\right), L_{p}(\mu, E)\right)$ and follow the ideas of the Rockafellar et al. (2006b) in order to represent the $V R F$ by "some kind of envelope generated by its sub-gradients".

Lemma 4.1. Suppose that $\mathcal{G}$ is a sub- $\sigma$-algebra of $\mathcal{F}, p<\infty$ and $F=\mathbb{R}$. If $D: L_{p}(\mu, E) \rightarrow \mathbb{R}$ is a real valued and continuous $\mathcal{G}$-deviation then there exists $\Delta \subset L_{q}\left(\mu, E^{*}\right)$ satisfying the following conditions:

a) $\Delta$ is convex and $\sigma\left(L_{q}\left(\mu, E^{*}\right), L_{p}(\mu, E)\right)$-compact. 
b) The equality

$$
D(y)=\operatorname{Max}\left\{-\int_{\Omega}\left\langle z^{*}(\omega), y(\omega)\right\rangle d \mu(\omega) ; z^{*} \in \Delta\right\}
$$

holds for every $y \in L_{p}(\mu, E)$.

Proof. Since $E^{*}$ satisfies the Radon-Nikodym property we have that $L_{q}\left(\mu, E^{*}\right)$ is the dual space of $L_{p}(\mu, E)$. Besides, if

$$
\Delta_{1}=\left\{z^{*} \in L_{q}\left(\mu, E^{*}\right) ; D(y) \geq \int_{\Omega}\left\langle z^{*}(\omega), y(\omega)\right\rangle d \mu(\omega), \forall y \in L_{p}(\mu, E)\right\}
$$

then it may be easily proved that $\Delta_{1}$ is convex and $\sigma\left(L_{q}\left(\mu, E^{*}\right), L_{p}(\mu, E)\right)$ closed. Furthermore, since $L_{q}\left(\mu, E^{*}\right)$ is the dual space of $L_{p}(\mu, E)$ and $D$ is continuous, Theorem 2.4.9 in Zalinescu (2002) implies that $\Delta_{1}$ is $\sigma\left(L_{q}\left(\mu, E^{*}\right), L_{p}(\mu, E)\right)$-compact, along with the equality

$$
D(y)=\operatorname{Sup}\left\{\int_{\Omega}\left\langle z^{*}(\omega), y(\omega)\right\rangle d \mu(\omega) ; z^{*} \in \Delta_{1}\right\}
$$

for every $y \in L_{p}(\mu, E)$. Hence, the result trivially follows if one takes $\Delta=$ $-\Delta_{1}$.

Remark 4.2. It is worth pointing out that every $z^{*} \in \Delta$ satisfies

$$
\mathbb{E}\left(z^{*} \mid \mathcal{G}\right)=0 .
$$

Indeed, Proposition 3.4.b) and the proof of Lemma 4.1. imply that

$$
-\int_{\Omega}\left\langle z^{*}, y_{0}\right\rangle d \mu \leq D\left(y_{0}\right)=0
$$

holds for every $y_{0} \in L_{p}\left(\mu_{\mathcal{G}}, E\right)$. Thus, if $-y_{0}$ replaces $y_{0}$ we have

$$
\int_{\Omega}\left\langle z^{*}, y_{0}\right\rangle d \mu=0
$$

Lemma 4.3. Suppose that $\mathcal{G}$ is a sub- $\sigma$-algebra of $\mathcal{F}, p<\infty, F=\mathbb{R}$ and $\Lambda: L_{p}\left(\mu_{\mathcal{G}}, E\right) \rightarrow \mathbb{R}$ is linear and continuous. If $\rho: L_{p}(\mu, E) \rightarrow \mathbb{R}$ is a real valued $(\Lambda, \mathcal{G})$-expectation bounded and continuous risk measure then there exist $\Delta \subset L_{q}\left(\mu, E^{*}\right)$ and $\Lambda_{\mathcal{G}} \in L_{q}\left(\mu_{\mathcal{G}}, E^{*}\right)$ satisfying Condition a) above and $\rho(y)=\operatorname{Max}\left\{-\int_{\Omega}\left\langle z^{*}(\omega), y(\omega)\right\rangle d \mu(\omega) ; z^{*} \in \Delta\right\}-\int_{\Omega}\left\langle\Lambda_{\mathcal{G}}(\omega), y(\omega)\right\rangle d \mu(\omega)$

holds for every $y \in L_{p}(\mu, E)$. Furthermore, $z^{*}(\omega)+\Lambda_{\mathcal{G}} \geq 0$ a.s. for every $z^{*} \in \Delta$ if and only if $\rho$ is $(\Lambda, \mathcal{G})$-coherent.

Proof. Proposition 3.5. and Expression ((4.1)) trivially imply the existence of $\Delta \subset L_{q}\left(\mu, E^{*}\right)$ satisfying Condition $\left.a\right)$ above and such that

$$
\rho(y)=\operatorname{Sup}\left\{-\int_{\Omega}\left\langle z^{*}(\omega), y(\omega)\right\rangle d \mu(\omega) ; z^{*} \in \Delta\right\}-\Lambda(\mathbb{E}(y \mid \mathcal{G}))
$$


for every $y \in L_{p}(\mu, E)$. Thus, if $\Lambda_{\mathcal{G}} \in L_{q}\left(\mu, E^{*}\right)$ is such that

$$
\int_{\Omega}\left\langle\Lambda_{\mathcal{G}}(\omega), y(\omega)\right\rangle d \mu(\omega)=\Lambda(\mathbb{E}(y \mid \mathcal{G}))
$$

for every $y \in L_{p}(\mu, E)$ then $((4.3))$ becomes obvious.

Suppose that $z^{*}(\omega)+\Lambda_{\mathcal{G}} \geq 0$ a.s. for every $z^{*} \in \Delta$. Then, take $y_{1}, y_{2} \in$ $L_{p}(\mu, E)$ with $y_{2} \geq y_{1}$ a.s. and

$-\int_{\Omega}\left\langle z^{*}(\omega)+\Lambda_{\mathcal{G}}(\omega), y_{1}(\omega)\right\rangle d \mu(\omega) \geq-\int_{\Omega}\left\langle z^{*}(\omega)+\Lambda_{\mathcal{G}}(\omega), y_{2}(\omega)\right\rangle d \mu(\omega)$

trivially holds. Thus, $\rho$ is $(\Lambda, \mathcal{G})$-coherent due to $((4.3))$.

Conversely, suppose that $\mu\left(\left\{\omega \in \Omega ; z^{*}(\omega)+\Lambda_{\mathcal{G}}(\omega) \notin E_{+}^{*}\right\}\right)>0$ for some $z^{*} \in \Delta$. Then, there exists $y \in L_{p}(\mu, E), y \geq 0$ a.s., such that

$$
\int_{\Omega}\left\langle z^{*}(\omega)+\Lambda_{\mathcal{G}}(\omega), y(\omega)\right\rangle d \mu(\omega)<0 .
$$

Hence, $((4.3))$ leads to $\rho(y)>0$, i.e., according to Proposition 3.4.a), $\rho(y)>$ $\rho(0)$, and $\rho$ is neither decreasing nor $(\Lambda, \mathcal{G})$-coherent.

Remark 4.4. Notice that $\left\{\Lambda_{\mathcal{G}}\right\}+\Delta$ may play the role of $\Delta$ in the latter lemma, in which case, we would obtain the existence of $\Delta$, convex and $\sigma\left(L_{q}\left(\mu, E^{*}\right), L_{p}(\mu, E)\right)$-compact, satisfying

$$
\rho(y)=\operatorname{Max}\left\{-\int_{\Omega}\left\langle z^{*}(\omega), y(\omega)\right\rangle d \mu(\omega) ; z^{*} \in \Delta\right\}
$$

for every $y \in L_{p}(\mu, E)$, and $\rho$ is $(\Lambda, \mathcal{G})$-coherent if and only if $z^{*}(\omega) \geq 0$ a.s. for every $z^{*} \in \Delta$. Notice also that $((4.2))$ leads to the equality

$$
\mathbb{E}\left(z^{*} \mid \mathcal{G}\right)=\Lambda_{\mathcal{G}}
$$

for every $z^{*} \in \Delta$.

Theorem 4.5 (Representation Theorem for Deviations). Suppose $\mathcal{G}$ is a sub$\sigma$-algebra of $\mathcal{F}$ and $p<\infty$. If $D: L_{p}(\mu, E) \rightarrow F$ is a continuous $\mathcal{G}$-deviation then for every $f^{*} \in F_{+}^{*}$ there exists $\Delta_{f^{*}} \subset L_{q}\left(\mu, E^{*}\right)$ satisfying the following conditions:

a) $\Delta_{f^{*}}$ is convex and $\sigma\left(L_{q}\left(\mu, E^{*}\right), L_{p}(\mu, E)\right)$-compact.

b) The equality

$$
f^{*} \circ D(y)=\operatorname{Max}\left\{-\int_{\Omega}\left\langle z^{*}(\omega), y(\omega)\right\rangle d \mu(\omega) ; z^{*} \in \Delta_{f^{*}}\right\}
$$

holds for every $y \in L_{p}(\mu, E)$.

c) $\mathbb{E}\left(z^{*} \mid \mathcal{G}\right)=0$ holds for every $z^{*} \in \Delta_{f^{*}}$.

Proof. It is a trivial consequence of Lemma 4.1. and Remark 4.2., if one takes into consideration that $f^{*} \circ D$ satisfies the properties of an $\mathbb{R}$-valued $\mathcal{G}$-deviation. 
Lemma 4.6. If $\mathcal{G}$ is a sub- $\sigma$-algebra of $\mathcal{F}, \Lambda: L_{p}\left(\mu_{\mathcal{G}}, E\right) \longrightarrow F$ is linear and continuous, and $\rho: L_{p}(\mu, E) \rightarrow F$ is a $(\Lambda, \mathcal{G})$-expectation bounded $V R F$, then $\rho$ is $(\Lambda, \mathcal{G})$-coherent if and only if $f^{*} \circ \rho$ is decreasing for every $f^{*} \in F_{+}^{*}$.

Proof. The result is clear if one bears in mind that $F$ is a Banach lattice and, thus, for $f_{1}, f_{2} \in F$ we have that $f_{1} \leq f_{2}$ if and only if $\left\langle f^{*}, f_{1}\right\rangle \leq\left\langle f^{*}, f_{2}\right\rangle$ for every $f^{*} \in F_{+}^{*}$.

Theorem 4.7 (Representation Theorem for Expectation Bounded VRF). Suppose that $\mathcal{G}$ is a sub- $\sigma$-algebra of $\mathcal{F}, \Lambda: L_{p}\left(\mu_{\mathcal{G}}, E\right) \longrightarrow F$ is linear and continuous and $p<\infty$. If $\rho: L_{p}(\mu, E) \rightarrow F$ is a $(\Lambda, \mathcal{G})$-expectation bounded and continuous $V R F$ then for every $f^{*} \in F_{+}^{*}$ there exist $\Delta_{f^{*}} \subset L_{q}\left(\mu, E^{*}\right)$ and $\Lambda_{\mathcal{G}} \in L_{q}\left(\mu_{\mathcal{G}}, E^{*}\right)$ satisfying the following conditions:

a) $\Delta_{f^{*}}$ is convex and $\sigma-\left(L_{q}\left(\mu, E^{*}\right), L_{p}(\mu, E)\right)$-compact.

b) The equality

$$
f^{*} \circ \rho(y)=\operatorname{Max}\left\{-\int_{\Omega}\left\langle z^{*}(\omega), y(\omega)\right\rangle d \mu(\omega) ; z^{*} \in \Delta_{f^{*}}\right\}
$$

holds for every $y \in L_{p}(\mu, E)$.

c) $\mathbb{E}\left(z^{*} \mid \mathcal{G}\right)=\Lambda_{\mathcal{G}}$ for every $z^{*} \in \Delta_{f^{*}}$.

Moreover, $\rho$ is $(\Lambda, \mathcal{G})$-coherent if and only if $z^{*} \geq 0$ a.s. for every $f^{*} \in F_{+}^{*}$ and every $z^{*} \in \Delta_{f^{*}}$.

Proof. It is a trivial consequence of Lemma 4.6. and Remark 4.4., if one takes into consideration that $f^{*} \circ \rho$ satisfies the properties of an $\mathbb{R}$-valued $\left(f^{*} \circ \Lambda, \mathcal{G}\right)$-expectation bounded risk measure.

\section{Examples and applications}

Example 1. It is worth pointing out that one can obtain vector risk functions by simultaneously considering several scalar risk functions. One might consider that this natural property should obviously hold, but recall that this is false for the alternative approach dealing with set-valued vector risk measures (Jouini et al., 2004, Cascos and Molchanov, 2007, Balbás and JiménezGuerra, 2010, or Hamel and Heyde, 2010).

More generally, consider the set of Banach lattices $E$ and $\left\{F_{j}\right\}_{j=1}^{n}$ where $E$ and $E^{*}$ satisfy the Radon-Nikodym property, the sub- $\sigma$-algebra $\mathcal{G}$ of $\mathcal{F}$, the family of continuous linear maps $\Lambda_{j}: L_{p}\left(\mu_{\mathcal{G}}, E\right) \rightarrow F_{j}$, and the risk functions $\rho_{j}: L_{p}(\mu, E) \rightarrow F_{j}, j=1,2, \ldots, n$. Take

$$
\rho: L_{p}(\mu, E) \rightarrow \prod_{j=n}^{n} F_{j}
$$


as usual. Then, if each $\rho_{j}$ is a $\mathcal{G}$-deviation so is $\rho$, and if each $\rho_{j}$ is $\left(\Lambda_{j}, \mathcal{G}\right)$ expectation bounded (respectively, $\left(\Lambda_{j}, \mathcal{G}\right)$-coherent) then $\rho$ is $(\Lambda, \mathcal{G})$-expectation bounded (respectively, $(\Lambda, \mathcal{G})$-coherent), where

$$
\Lambda\left(y_{0}\right)=\left(\Lambda_{j}\left(y_{0}\right)\right)_{j=1}^{n} \in \prod_{j=n}^{n} F_{j}
$$

for every $y_{0} \in L_{p}\left(\mu_{\mathcal{G}}, E\right)$.

Example 2. Jouini et al. (2004) provided interesting situations making it quite convenient to consider vector-valued random variables to represent final wealths (or pay-offs) and risk levels (see also Cascos and Molchanov, 2007). For instance, if a portfolio were diversified amongst several currencies and transaction costs made it inefficient to compensate possible losses in a given currency with those profits generated in the remaining ones, then we could take $E=\mathbb{R}^{n}$ and $F=\mathbb{R}^{m}$ with $m \leq n$. Then, $y \in L_{p}(\mu, E)$ would indicate the pay-off of each sub-portfolio associated with the corresponding currency, and $\rho(y)$ could indicate the capital requirement to overcome the risk, currency by currency. Of course, $m$ may be less than $n$ because some currencies may be liquid enough so as to accept compensations, and $\mathcal{G}=\{\varnothing, \Omega\}$.

The arguments above also apply in different situations non-necessarily related to currencies. Mainly, one needs a global portfolio that may be divided into sub-portfolios in such a way that it is expensive to compensate among them all due to imperfections.

Example 3.(Dynamic risk measures) Dynamic risk measures are very important in Actuarial and Financial Mathematics because prices, risks and strategies evolve in a stochastic framework. There are several approaches dealing with the notion of dynamic measure of risk, though the most usual one is that in Frittelli and Rosazza Gianin (2004), Cheridito et al. (2005) or Roorda and Schumacher (2011), among others. Thus, consider the closed interval $[0, T]$ representing a time period and a set $\mathcal{T} \subset[0, T]$ representing the trading dates and such that $\{0, T\} \subset \mathcal{T}$. The arrival of information is given by the filtration $\left(\mathcal{G}_{t}\right)_{t \in \mathcal{T}}$ such that $\mathcal{G}_{0}=\{\varnothing, \Omega\}$ and $\mathcal{G}_{T}=\mathcal{F}$. For every adapted stochastic process $\left(S_{t}\right)_{t \in \mathcal{T}}$ such that $S_{t} \in L_{2}\left(\mu_{\mathcal{G}_{t}}, \mathbb{R}\right)$, usually representing an agent wealth (or loss) at every date $t \in \mathcal{T}$, one can fix $s<t \in \mathcal{T}$ and then, define a risk function $\rho_{(s, t)}: L_{2}\left(\mu_{\mathcal{G}_{t}}, \mathbb{R}\right) \rightarrow L_{2}\left(\mu_{\mathcal{G}_{s}}, \mathbb{R}\right)$. Actually the system $\left(\rho_{(s, t)}\right)_{s<t \in \mathcal{T}}$ is a dynamic risk measure, and the analysis fits in our much more general approach if one takes $E=\mathbb{R}, F=L_{2}\left(\mu_{\mathcal{G}}, \mathbb{R}\right), \mathcal{G}=\mathcal{G}_{s}$, and $\Lambda: L_{2}\left(\mu_{\mathcal{G} s}, \mathbb{R}\right) \rightarrow L_{2}\left(\mu_{\mathcal{G} s}, \mathbb{R}\right)$ given by the identity map. Thus, our general definitions of Section 3 and the Representation Theorems of Section 4 apply for dynamic measures of risk. Notice finally that the role of $E=\mathbb{R}$ may be plaid by other vector space, and consequently the approach of this paper applies for both scalar and vector dynamic risk measures. As far as we know, the notion of vector dynamic risk measure had not been introduced yet in the literature. 
Example 4.(Optimizing vector risk functions) The optimization of scalar measures of risk is a very important problem in Actuarial and Financial Mathematics because many practical decisions must be optimal. For instance, Benati (2003) and Konno et al. (2005) study portfolio choice problems, Schweizer (1995) and Nakano (2004) deal with pricing issues, Balbás et al. (2009) analyze optimal reinsurance problems, and Rockafellar et al. (2006a) and Balbás et al. (2010b) deal with market equilibrium topics.

In general, the minimization of scalar risk measures is complex in practice because risk measures are not differentiable functions, and the usual Lagrangian linked methods do not apply. Thus, most of the papers above must develop special methods that solve their concrete problem. Nevertheless, there are recent papers whose focus is on the minimization of risk, and they provide general optimization techniques (Balbás et al., 2010a, among others). The representation theorems play a crucial role in the development of these general methodologies, since they allow us to find equivalent differentiable optimization problems.

Vector optimization problems are also usual in practical applications. If they are convex, which always holds if one minimizes vector risk measures, then, the most important ways to solve them are the scalarization method and the balance space approach (Galperin, 1997). In both cases, the Representation Theorems of Section 4 permit us to extend the findings of Balbás et al. $(2010 a)$, in such a way that the minimization of vector risks become a differentiable problem, and then, standard Lagrangian linked and saddle point linked necessary and sufficient optimality conditions may be given. We will not present a detailed analysis because this is a straightforward extension of Balbás et al. (2010a), if one bears in mind Theorems 4.5. and 4.7.

\section{Conclusions}

The paper has introduced a new notion of vector risk function and concepts such as vector deviation, vector expectation bounded risk measure or vector coherent risk measure. Relationships amongst them have been analyzed. In this sense, the generalized vector risk functions may be used to provide initial capital requirements as well as to deal with most of the classical topics (pricing, hedging, portfolio choice, etc.). The link with dynamic risk functions or vector risk functions studied in previous literature has been discussed, and it has been pointed out that this new approach simplifies many theoretical and practical problems, since we do not deal with set-valued risks. On the contrary, the risk of every (vector) pay-off is a single vector. Practical examples have been illustrated and sub-gradient linked representation theorems have been given. 


\section{References}

[1] P. Artzner, F. Delbaen, J.M. Eber and D. Heath, Coherent measures of risk. Mathematical Finance 9 (1999), 203-228.

[2] A. Balbás, B. Balbás and R. Balbás, Minimizing measures of risk by saddle point conditions. Journal of Computational and Applied Mathematics 234 (2010a), 2924-2931.

[3] A. Balbás, B. Balbás and R. Balbás, CAPM and APT-like models with risk measures. Journal of Banking \& Finance 34 (2010b), 1166-1174.

[4] A. Balbás, Balbás, B., Heras, A., Optimal reinsurance with general risk measures. Insurance: Mathematics and Economics 44 (2009), 374-384.

[5] A. Balbás and P. Jiménez-Guerra, Set-valued vector risk functions. The Journal of Financial Decision Making 6 (2010), 41-49.

[6] S. Benati, The optimal portfolio problem with coherent risk measure constraints. European Journal of Operational Research 150 (2003), 572-584.

[7] I. Cascos. and I. Molchanov, Multivariate risks and depth trimmed regions. Finance \& Stochastics 11 (2007), 373-397.

[8] P. Cheridito, F. Delbaen, and M. Kupper, M., Coherent and convex monetary risk measures for unbounded càdlàg processes. Finance \& Stochastics 9 (2005), 369-387.

[9] J. Diestel and J.J. Uhl, Vector measures. American Mathematical Society, Providence, Rhode Island (1977).

[10] H. Föllmer and A. Schied, Convex measures of risk and trading constraints. Finance \& Stochastics 6 (2002), 429-447.

[11] M. Frittelli and E. Rosazza Gianin, Dynamic convex risk measures. In: Szegö, G. (Ed.), Risk Measures for the 21st Century. Wiley, New York, (2004) pp. 227-248.

[12] E.A. Galperin, Pareto analysis vis-à-vis balance apace approach in multiobjective global optimization. Journal of Optimization Theory and Applications 93 (1997), 3, 533-545.

[13] M. Goovaerts, R. Kaas, J. Dhaene and Q. Tang, A new classes of consistent risk measures. Insurance: Mathematics and Economics 34 (2004), 505-516.

[14] A. Hamel and F. Heyde, Duality for set-valued measures of risk. SIAM Journal of Financial Mathematics 1 (2010), 66-95.

[15] E. Jouini, M. Meddeb and N. Touci, Vector-valued coherent risk measures. Finance \& Stochastics 8 (2004), 531-552.

[16] H. Konno, K. Akishino and R. Yamamoto, Optimization of a long-short portfolio under non-convex transaction costs. Computational Optimization and Applications 32 (2005), 115-132.

[17] P. Meyer-Nieberg, Banach lattices. Springer-Verlag. New York (1991).

[18] Y. Nakano, Efficient hedging with coherent risk measure. Journal of Mathematical Analysis and Applications 293 (2004), 345-354.

[19] W. Ogryczak, and A. Ruszczynski, From stochastic dominance to mean risk models: Semideviations and risk measures. European Journal of Operational Research 116 (1999), 33-50.

[20] B. Roorda and J.M. Schumacher, The strictest common relaxation of a family of risk measures. Insurance: Mathematics and Economics 48 (2011), 29-34. 
[21] R.T. Rockafellar, S. Uryasev and M. Zabarankin, Optimality conditions in portfolio analysis with general deviations measures. Mathematical Programming, Ser. B, 108 (2006a), 515-540.

[22] R.T. Rockafellar, S. Uryasev and M. Zabarankin, Generalized deviations in risk analysis. Finance \& Stochastics 10 (2006b), 51-74.

[23] M. Schweizer, Variance-optimal hedging in discrete time. Mathematics of Operations Research 20 (1995), 1, 1-32.

[24] C. Zalinescu, Convex analysis in general vector spaces. World Scientific Publishing Co,(2002).

Alejandro Balbás

University Carlos III of Madrid.

CL. Madrid 126.

28903 Getafe, (Madrid)

Spain

e-mail: alejandro.balbas@uc3m.es

Raquel Balbás

University Complutense of Madrid.

Department of Actuarial and Financial Economics.

Somosaguas Campus.

28223 Pozuelo de Alarcón, (Madrid)

Spain.

e-mail: raquel.balbas@ccee.ucm.es

Pedro Jiménez-Guerra

Spanish Open University.

Department of Fundamental Mathematics.

CL. Senda del Rey, 9.

28040 Madrid

Spain.

e-mail: pjimenez@mat.uned.es 